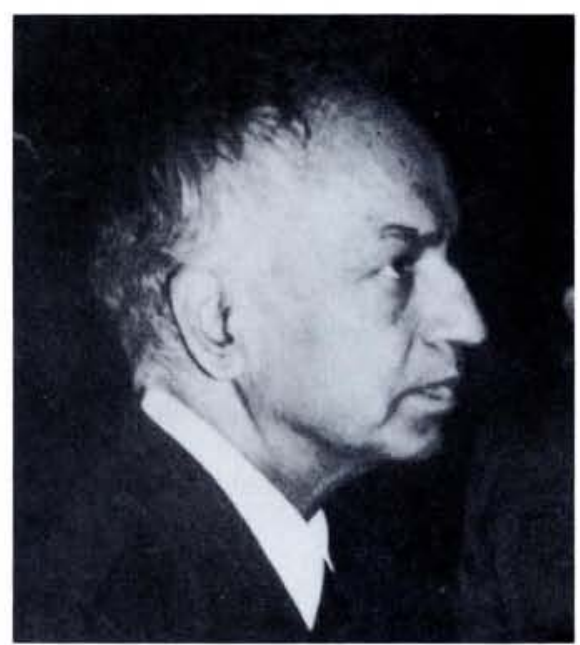

Subrahmanyan Chandrasekhar

\section{In Honour of the Stars and of the Universe}

\author{
Jean Audouze, Paris
}

(Directeur

de l'Institut d'Astrophysique du CNRS)

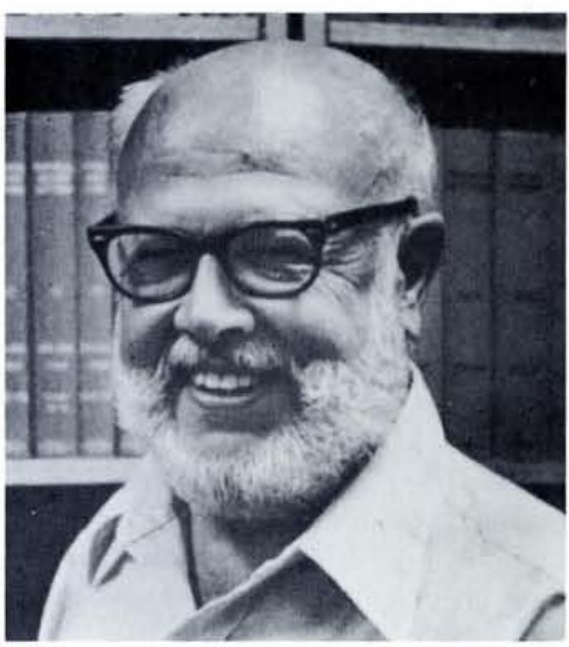

William A. Fowler
Two most distinguished astrophysicists, Professors Subrahmanyan Chandrasekhar from the Enrico Fermi Institute of the University of Chicago and William A. Fowler from the W.K. Kellogg Radiation Laboratory of the California Institute of Technology have been awarded the 1983 Nobel Prize in Physics for their outstanding contributions in stellar astrophysics.

Chandrasekhar was born in Lahore in 1910, went as a graduate student to Cambridge in 1930 and finally joined the University of Chicago in 1935. Among his many theoretical works of the utmost importance, he has performed a very thorough analysis of the random walk motion of particles in fluids and more recently an analysis of the physics of black holes and their evolution. But the 1983 Nobel Prize has certainly been awarded to him for a remarkable and pivotal discovery in stellar astrophysics: during the thirties, Chandrasekhar was able to establish an important stellar mass limit that separated two domains wherein the stellar evolution is quite different.

1) If the mass of a star is below the precise limit of 1.44 solar masses (which is referred to as the Chandrasekhar limit), the star after being a red giant loses its envelope and becomes a planetary nebula; after that the remaining star contracts and becomes a white dwarf. A white dwarf is characterized by a large surface temperature $(\gtrsim 50000 \mathrm{~K}$, at least at the beginning of the stage) and by a very small radius in comparison with its mass. When the Sun, in about 5000 million years, becomes a white dwarf, its radius will be only $1 \%$ of its present value (i.e. comparable to that of the Earth today). Densities as large as $10^{11} \mathrm{~kg} / \mathrm{m}^{3}$ are reached in the central regions of these objects. The behaviour of the matter at such high densities is similar to that of a piece of steel: the pressure does not depend on the temperature which is a consequence of the degeneracy of the electrons in the gas. When a low mass star has reached this stage it can be considered as a fossil of the stellar evolution: after the exhaustion of the contraction energy, the white dwarf becomes a black dwarf i.e. an invisible object which contributes only to the mass density of the Universe.

2) If the mass of a star is larger than the Chandrasekhar limit, its evolution is radically different. Such stars after the red giant stage do not expand their envelope gently like the planetary nebulae; instead they undergo a violent and spectacular explosion known as the supernova phenomenon. A very famous example of it is the explosion forming the Crab nebula which occurred in 1043 AD. While the external layers explode, the internal regions contract to become a neutron star. This occurs when the density becomes as large as $10^{15} \mathrm{~kg} / \mathrm{m}^{3}$ : electrons are captured by protons to become neutrons which are then stable. The typical radius of neutron stars is a few $\mathrm{km}$ for masses of about two solar masses. Neutron stars can be detected in different wavelengths but especially in radio by the pulsar phenomenon which was discovered by A. Hewish (Nobel Prize in physics in 1975) and J. Bell.

The work which has been performed by Willy Fowler and his many associates, students, friends and collaborators is quite complementary to that of Chandrasekhar. Fowler has given the letters of nobility to one of the most active branches of astrophysics, i.e. nuclear astrophysics. In a very influential paper which appeared in 1957 in Reviews of Modern Physics, written in collaboration with the British astrophysicists Fred Hoyle, Margaret and Geoffrey Burbidge, the basis of nuclear astrophysics was clearly and deeply established. The classification of the different nucleosynthetic processes occurring in stars land also during the Big Bang and those induced by cosmic rays) proposed in that paper (and also independently the same year by Al Cameron in his Chalk River report) is still in use. In both papers one finds reference to the hydrogen burning which occurs in main sequence stars; to the helium, carbon, and oxygen burning which occurs in giants and supergiant stars; to the photodisintegration of the iron peak elements which should trigger the explosion of the massive supernovae; to the slow and the rapid neutron absorption processes which account for the formation of the heavy elements; to the p process which tries to explain the rarest stable isotopes of heavy elements in which the nuclei are relatively rich in protons; finally what they called the $x$ process (for unknown), responsible for the formation of the lightest rare nuclear species like deuterium, lithium, beryllium and boron. We know now that deuterium and lithium - 7 should be synthetized together with helium -3 and -4 by the Big Bang nucleosynthesis, while ${ }^{6} \mathrm{Li}$, ${ }^{9} \mathrm{Be},{ }^{10} \mathrm{~B}$, and ${ }^{11} \mathrm{~B}$ should be formed by the interaction between the galactic cosmic rays and the interstellar medium.

The relation between the contributions of Chandrasekhar and Fowler is obvious. Chandrasekhar fixed the domain 
in which most of the nuclear astrophysics processes analyzed by Fowler take place. The explosions of supernovae which have been thoroughly examined by Fowler and Hoyle and which concern only massive stars (as stated by Chandrasekhar) are the astrophysical objects where most of the nucleosynthetic processes take place, the by-products of which are able to enrich the interstellar medium.

Willy Fowler was born in Ohio in 1911 and went to Caltech in Pasadena California as a graduate student in nuclear physics in 1933. He then climbed through the professorial ranks from 1939 until 1970 when he became Institute Professor of Physics. His career started as an experimentalist in nuclear physics under the guidance of $\mathrm{C}$. Lauritsen, but after World War II he took advantage of his encounter, collaboration and friendship with Fred Hoyle to become interested in the applications of nuclear physics to astrophysics. The number of his personal contributions to this field and the number of scientists who have personally benefited from his guidance, collaboration and advice are considerable.

Among his notable contributions, besides the monumental $\mathrm{B}^{2} \mathrm{FH}$ paper, one can point out his work on various aspects of stellar nucleosynthesis and, in particular, his papers on the $s$ and $r$ process (performed in collaboration with D.D. Clayton and P. Seeger); those on the causes of the explosions of massive supernovae based upon Fe photodisintegration (performed in collaboration with Hoyle); his many fundamental contributions on the determinations of nuclear reaction rates of astrophysical interest; his work on nucleocosmochronology (again in collaboration with Hoyle). The quite barbaric word nucleocosmochronology designates the method through which one can determine the age of the Universe or of the solar system by analyzing the abundances of the nuclear species which come from the decay of long lived radioactive nuclei like ${ }^{235} \mathrm{U},{ }^{238} \mathrm{U},{ }^{232} \mathrm{Th},{ }^{244} \mathrm{Pu},{ }^{129} \mathrm{I}$ etc. By such techniques, Fowler and Hoyle determined the age of the Universe to be about $10^{9}$ years, consistent with what can be deduced from the recession of the galaxies (namely by inversing the Hubble constant) or from the age of the globular clusters. Finally, one may note without surprise that many significant discoveries concerning the isotope anomalies in meteorites have been made in the so-called "Lunatic Asylum" of Caltech (under the direction of G.J. Wasserburg). Such discoveries like those which concern ${ }^{26} \mathrm{Al}$ and the isotopic composition of elements such as $\mathrm{Ca}$, $\mathrm{Ti}, \mathrm{Nd}, \mathrm{Sm}$, etc. have been, indirectly at least, inspired by Fowler. Thanks to him, we know that we are constituted from the ashes of supernovae which exploded some 5000 millions years ago or before.

There are several reasons why the work of Fowler has taken more room to describe than the incisive contributions of Chandrasekhar. First, it takes more words to describe the results of nuclear astrophysics than the thermodynamics of the stars. Second, the work which has been achieved and/or inspired by Fowler has come from the efforts of many individuals, both theoreticians and experimentalists. This shows how diverse exceptional careers can be - either individualistic like that of Chandrasekhar or deliberately oriented towards many collaborations like that of Fowler. As Willy Fowler was planning to mention in his address in Stockholm which will be devoted to the helium burning process, most of the significant discoveries which have been made concerning this process have come directly or indirectly from the team work of the Kellogg Radiation Laboratory of Caltech; it is because the scientists of this laboratory established the instability of nuclei with atomic masses 5 and 8 that E.E. Salpecter and then Hoyle made their ingenious and important contributions on the twostep mechanism that leads to the transformation of ${ }^{4} \mathrm{He}$ into ${ }^{12} \mathrm{C}$.

Finally I should add a personal reason which is my own tribute to Willy Fowler. I was fortunate to work as a post doctoral fellow for about two years in $1971-1973$ at the Kellogg Radiation Laboratory under his sponsorship. I continue to take every possible opportunity to pursue my visits to this more than excellent centre of research and to enjoy his enthusiasm and friendship.

By awarding the 1983 Nobel Prize in physics to these two prominent astrophysicists, I feel sure that the Nobel committee wanted to recognize the importance of research in astrophysics in general and in stellar astrophysics in particular. Astrophysics is indeed one of the domains of science where a lot of very important discoveries are presently being made. About twenty years ago, M. Schmidt was just discovering the quasars on which Fowler made important theoretical contributions; the pulsars were unknown, all the space adventure was just starting, the black body radiation which constitutes one of the major arguments in favour of the Big Bang theory was discovered by A. Penzias and
R. Wilson (also Nobel Prize winners) only in 1965. With the advent of the many important space missions like Voyager and IRAS and, in a near future, the Space Telescope, the astrophysicists should obtain a wealth of invaluable data which will allow them to unravel further the mysteries of our Universe.

The award of 1983 concerns theoretical astrophysics and the application of microphysics to astrophysics. These observations will only be understood if the astrophysicists master the most advanced theories dealing not only with the thermodynamics of fluid dynamics but also with particle physics. More and more, joint effort from the particle physics and the astrophysics communities will try to solve problems such as the evolution of the early phases of the Universe, the physics of the neutrinos... It may not just be fortuitous that CERN and ESO jointly organized from 21-25 November 1983 an international symposium on the relations between astrophysics and particle physics and that the General Director of the French Centre National de la Recherche Scientifique is launching a new interdisciplinary programme concerning this field.

The award to these very talented, productive and enthusiastic scientists exhibits the importance of theoretical and nuclear astrophysics in understanding the evolution of stars which constitutes a basic problem in astrophysics. For Professors Subrahmanyan Chandrasekhar and William A. Fowler this prize is the coronation, not only of exceptional scientific achievements, it is also the recognition by the international community of the very important role they have played in the whole scientific endeavour. One should recall that Professor Chandrasekhar was the Managing Editor of the Astrophysical Journal during twenty years from 1952 to 1972 while Professor Fowler, besides his important contribution to the defense of his country during World War II, has served on many important committees and occupied many functions such as the presidences of the American Physical Society and the National Academy of Sciences. Finally I should like to point out the unusual dynamism of these senior scientists who both still pursue very actively to-day their scientific work: the book on black holes by Chandrasekhar appeared this year while Fowler has many papers on nuclear astrophysics either in press or in preparation. The whole astrophysical community is therefore much honoured by the prestigious award so well deserved by these two exceptional men. 